www.jmscr.igmpublication.org

Impact Factor (SJIF): 6.379

Index Copernicus Value: 71.58

ISSN (e)-2347-176x ISSN (p) 2455-0450

crossref DOI: https://dx.doi.org/10.18535/jmscr/v6i6.32

Journal Of Medical Science And Clinical Research

IGM Publication

An Official Publication of IGM Publication

\title{
Estimation of Quality of Life (QOL) in Patients of Tuberculosis Attending- A Tertiary Medical Centre
}

\author{
Authors \\ Mukesh Tiwari, Dr Santosh Kumar, Dr Gajendra Vikram Singh, Dr Vishal Sinha \\ Dr Gyan Prakash \\ S.N.M.C. Agra (U.P.), India
}

\begin{abstract}
Aims and Objective: The aims of study to compare the quality of life (QOL) in patients of Tuberculosis (TB) depending on category and duration of treatment.

Methods: We evaluated, 240 patients 18 to 60 years of age registered under Revised National Tuberculosis Control Program Directly Observed Treatment Short Course (RNTCP DOTS) between April, 2015 to March, 2016 presented to Department of Tuberculosis \& Chest Diseases, S.N. Medical College, Agra. We will conduct an observational prospective periodic study to assess quality of life by using World Health Organization Quality of Life-BREF Assessment Instrument (WHOQOL-BREF, Hindi version) at 10-15 days and 55-60 days after the registration under RNTCP DOTS.

Results: At 10-15days out of the 240 patients of tuberculosis, 59 patients were taking treatment of category I, 106 patients were taking treatment of category II, and 75 patients were taking treatment of category IV. The Mean $\pm S D$ of quality of life of patients were taking treatment of category I are 59.03 \pm 7.51 , patients were taking treatment of category II are 53.05 \pm 7.25 and patients were taking treatment of category IV are 52.80 \pm 8.28 . Whereas at 55-60days out of the 217 patients, , 59 patients were taking treatment of category I, 92 patients were taking treatment of category II, and 66 patients were taking treatment of category IV. The Mean $\pm S D$ of quality of life of patients were taking treatment of category I are $75.9 \pm 8.88$, patients were taking treatment of category II are $73.28 \pm 8.60$ and patients were taking treatment of category IV are 73.64 \pm 10.08 . Thus quality of life of patients significantly ( $p$-value-<0.0001) improved between different category of treatment on 55-60days as compare to 10-15 days.

Conclusion: Patients with taking treatment of category IV had lower mean scores than taking treatment of category I and II for overall QOL. Quality Of Life in patients of tuberculosis significantly improves at 5560days as compare to 10-15days after registration under RNTCP DOTS.

Keyword: Tuberculosis, Quality of Life.
\end{abstract}

\section{Introduction}

Tuberculosis (TB) is a leading cause of global morbidity, yet there is limited information regarding its impact on quality of life and health status. This is surprising given the implications for patient care, the evaluation of novel treatments or preventative strategies, and also health policy. The World Health Organization (WHO) defined quality of life as “individuals' perceptions of their positions in life in the context of the culture and value systems in which they live and in relation to their goals, expectations, standards and 
concerns"1. A patient with tuberculosis faces several physiological, psychological, financial and social problems. These problems have a great impact on the well-being of the patient and impair the quality of life of the patient suffering from tuberculosis. There are numerous aspects of active Tuberculosis disease that may lead to a reduction in Health Related Quality of life (HRQOL). Standard anti-Tubercular treatment requires prolonged therapy (at least 6-8 months) with multiple potentially toxic drugs that can lead to adverse reactions in a significant number of patients $^{44}$. There is considerable social stigma associated with active Tuberculosis, leaving the individuals feeling neglected and isolated from their friends and families ${ }^{2,3}$ because they are perceived as sources of infection leading to a long-term impairment in patients' psychosocial well-being ${ }^{4,5}$.

\section{Material and Method}

We evaluated, 240 patients 18 to 60 years of age registered under Revised National Tuberculosis Control Program Directly Observed Treatment Short Course (RNTCP DOTS) between April, 2015 to March, 2016 presented to Department of Tuberculosis \& Chest Diseases, S.N. Medical College, Agra. We will conduct an observational prospective periodic study to assess quality of life by using World Health Organization Quality of Life-BREF Assessment Instrument ${ }^{6}$ (WHOQOLBREF, Hindi version) at 10-15 days and 55-60 days after the registration under RNTCP DOTS.At 10-15 days 240 patients enrolled into study, whereas on fallow up study at 55-60 days out of 240 patients enrolled into our study, evaluation of 23 patients were not fallow up at RNTCP DOTS centre and only 217 patient were further evaluated.

\section{Criteria for Inclusion}

- All patients registered under RNTCP taking DOTS for tuberculosis at Department of Tuberculosis and Chest diseases, S. N. Medical College, Agra from April 2015 to March 2016.
- Age of patients to be between 18 to 60 years.

- Patients who are able to give informed consent.

\section{Criteria for Exclusion}

- All patients registered under RNTCP taking DOTS for tuberculosis at Department of TB and Chest diseases, S. N. Medical College, Agra before April 2015 and after March 2016.

- Patients who are not able to give informed consent.

- Patients not in a physical or mental state in which they can comprehend the questions asked or who are not capable of giving a valid response.

- Any patient with co-morbidities which affects occurrence or severity of tuberculosis.

\section{Results}

At 10-15days out of the 240 patients of tuberculosis, 59 patients were taking treatment of category I, 106 patients were taking treatment of category II, and 75 patients were taking treatment of category IV. The Mean \pm SD of quality of life of patients were taking treatment of category I are $59.03 \pm 7.51$, patients were taking treatment of category II are 53.05 \pm 7.25 and patients were taking treatment of category IV are 52.80 \pm 8.28 .

Whereas at 55-60days out of the 217 patients, , 59 patients were taking treatment of category I, 92 patients were taking treatment of category II, and 66 patients were taking treatment of category IV. The Mean \pm SD of quality of life of patients were taking treatment of category I are $75.9 \pm 8.88$, patients were taking treatment of category II are $73.28 \pm 8.60$ and patients were taking treatment of category IV are $73.64 \pm 10.08$.

Thus quality of life of patients significantly ( $\mathrm{p}$ value-<0.0001) improved between different category of treatment on 55-60days as compare to 10-15 days. 
Table 1: Category of Treatment and Whoqol-Bref

\begin{tabular}{|c|c|c|c|c|c|c|}
\hline \multirow{2}{*}{$\begin{array}{l}\text { Category of } \\
\text { treatment }\end{array}$} & \multicolumn{2}{|c|}{ WHOQOL-BREF (10-15days) } & \multicolumn{2}{|c|}{ WHOQOL-BREF(55-60days) } & \multirow{2}{*}{$|\mathrm{t}|$-value } & \multirow{2}{*}{$\mathrm{P}$-value } \\
\hline & No. & Mean \pm SD & No. & Mean \pm SD. & & \\
\hline I & 59 & $59.03 \pm 7.51$ & 59 & $75.9 \pm 8.88$ & 11.1421 & $<0.0001$ \\
\hline II & 106 & $53.05 \pm 7.25$ & 92 & $73.28 \pm 8.60$ & 17.7443 & $<0.0001$ \\
\hline \multirow[t]{2}{*}{ IV } & 75 & $52.80 \pm 8.28$ & 66 & $73.64 \pm 10.08$ & 13.3044 & $<0.0001$ \\
\hline & 240 & & 217 & & & \\
\hline
\end{tabular}

\section{Discussion}

Measurement of the WHOQOL-BREF adds a new dimension to the evaluation of Tuberculosis programs. With the development of effective treatment strategies where mortality is likely to be minimal, the focus of TB management has shifted to the reduction of illness-related morbidity. In this context, this study highlights the healthrelated quality of life among tuberculosis patients attending the TB clinic. The findings of a study conducted in 2009, to measure health-related QOL in TB showed that TB had a substantial and encompassing impact on patients' QOL. Overall, the anti-TB treatment had a positive effect on improving patients' QOL; their physical health tended to recover more quickly than the mental well-being. ${ }^{7}$ Another study of the impact of TB on the QOL and the effect after treatment with DOTS conducted in Delhi in 2009 using a questionnaire to assess the QOL showed that patients with TB had significantly lower mean scores than controls for overall QOL. In 2010, a study done to assess the impact of TB and its treatment on patients' health status showed that TB patients suffered from significantly diminished health-related QOL at diagnosis. Although treatment significantly improved the status of patients' health within two months, scores for many domains remained below the United Kingdom norm scores. This emphasizes the importance of a holistic approach to care and should inform the evaluation of future interventions. $^{8}$

\section{Conclusion}

The findings of this study indicate that patients with drug resistant tuberculosis had significantly lower mean rank scores than patients who are non- drug resistant for overall QOL. Quality Of Life in patients of tuberculosis significantly improves at 55-60days as compare to 10-15days after registration under RNTCP DOTS. Based on these results, the following are recommended. First, greater attention should be given to the quality of life of TB patients. Secondly, educational materials and information for dissemination by the media should be developed (Web-and print-based) to help raise public awareness of the prevention and control of TB.

\section{Acknowledgements and disclosures: none Statement of interests: none}

\section{Bibliography}

1. The World Health Organization Quality of Life Assessment

(WHOQOL): development and general psychometric properties. Soc Sci Med 1998;46:1569-85.

2. Yamada S, Caballero J, Matsunage DS. Attitudes regarding tuberculosis in immigrants from the Philippines to the United States. Fam Med 1999;31:477-82.

3. Long NH, Johansson E, Diwan VK. Fear and social isolation as consequences of tuberculosis in Vietnam: a gender analysis. Health Policy 2001;58:69-81.

4. Liefooghe R, Michiels N, Habib S, Moran MB, De Muynck A. Perception and social consequences of tuberculosis: a focus group study of tuberculosis patients in Sialkot, Pakistan. Soc Sci Med 2005;41:1685-92.

5. Marra CA, Marra F, Cox VC, Palepu A, Fitzgerald JM. Factors influencing quality 
of life in patients with active tuberculosis. Health Qual Life Outcomes 2004;20:58.

6. The World Health Organization Quality of Life (WHOQOL)-BREF (C) World Health Organization 2004.

7. N. Guo, F. Marra, C.A. Marra Measuring health-related quality of life in tuberculosis: a systemic reviewHealth Qual Life Outcomes, 7 (2009 February 18), p. 14.

8. M.E. Kruijshaar, M. Lipman, M.L. EssinkBot, S. Lozewicz, D. Creer, S. Dart, H. Maguire, I. AbubakarHealth status of UK patients with active tuberculosis Int J Tuberc Lung Dis, 14 (3) (2010 Mar), pp. 296-302. 